\title{
Impact of the Publication of Chinese Treatment Guideline on the Initial Therapy in Parkinson's Disease in Beijing
}

\author{
Haitian Nan, Kai Li, Shuhua Li, Wen Su and Haibo Chen* \\ Department of Neurology, Beijing Hospital, China \\ *Corresponding author: Haibo Chen, Department of Neurology, Beijing Hospital, China
}

Received: 制 July 31, 2019

Published: 監August 08, 2019

\begin{abstract}
Background: The Guideline for Management of Parkinson's Disease in China was published in 2006 to standardize Parkinson's disease treatment. Our objective was to compare the initial PD treatment and their accordance with the recommendations before and after the guideline publication.

Methods: We identified 136 PD patients as part of a hospital-based study in Beijing, and compared the prescriptions of Dopamine Agonists (DA) and levodopa (LD) as initial therapy to evaluate the impact of the publication of Chinese guideline on the therapy of PD.

Results: We found that the publication of the guideline resulted in no difference in initial treatment of PD patients $>65$ years. In patients $<65$ years, the prescription of DA was significantly increased after the publication of the guideline. There were no significant differences in initial treatment between patients treated in hospitals of different levels or patients with different types of insurance.
\end{abstract}

Conclusion: The guideline promoted DA utilization as initial treatment in young patients.

Keywords: Parkinson Disease; Initial Therapy; Treatment Guideline; Dopamine Agonist; Beijing

\section{Introduction}

The most commonly used antiparkinsonian drugs in China include: anticholinergics (trihexyphenidyl), Amantadine, Levodopa (LD), Dopamine Agonists (DA), Monoamine Oxidase (MAO)-B inhibitors, Catechol-O-Methyltransferase (COMT) inhibitors. LD is the most effective antiparkinsonian drug [1], but its long-term use is associated with motor complications [2]. DA poses less risk of long-term motor complications than LD [3] but is less effective. The challenge during initial PD stages is to control motor symptoms and restore quality of life without increasing the risk of long-term complications. In July 2006, the Guideline for Management of Parkinson's Disease in China was published. Our objective was to compare the initial PD treatment and their accordance with the recommendations before and after the guideline's publication.

\section{Subjects and Methods}

\section{Participants}

One hundred and thirty-six PD patients were enrolled from two hospitals through out-patient department of neurology: Beijing
Hospital of the Ministry of Health (class 3-A hospital) and Pinggu traditional Chinese medical hospital (class 2-A hospital). Patients were examined by movement disorders specialists and inquired about the date of symptoms onset, initial treatment, and age at treatment initiation. They were invited to provide all their PD drug prescriptions. Diagnosis was established using standardized criteria [4]. All subjects had at least one prescription of LD, COMT inhibitor (entacapone), Amantadine, anticholinergics (trihexyphenidyl), DA (pramipexole and piribedil), or MAO-B inhibitors (selegiline and rasagiline). Additional inclusion criteria were: disease duration $>1$ month and Hoehn-Yahr stage less than II.

\section{Chinese Guideline on the Therapy of Pd}

The guideline on PD treatment in China was proposed by the Chinese Movement Disorders and Parkinson's Disease Society. The experts in the study group are all well-recognized on treating PD and related motor disorders. The guideline is a result of a comprehensive review of existing literature and the actual situation in China. The first edition was published in June 2006 in Chinese 
Journal of Neurology which aimed at making recommendations regarding PD diagnosis and treatment and is widely accessed by both clinical physicians and scientific researchers [5]. The published guideline includes the time of drug utilization and the choice of initial medications in the treatment of early symptoms of Parkinson's disease. And it was the first time in China to recommend standard Parkinson's disease treatment. The guideline suggests that DA is the mainstay choice followed by selegiline with or without vitamin $\mathrm{E}$ for patients younger than age 65 years without cognitive impairment. When the medications mentioned above fail to improve the symptoms, a combination therapy with compound LD and COMT inhibitor is required. However, for some patients with diminished cognitive function or needing remarkable improvement, compound LD can be the initial therapy. For old $(\geq 65$ years) patients, compound LD should be the initial choice, if needed, it can be combined with DA, MAO-B inhibitor or COMT inhibitor. In 2001, America published the third edition of the guidelines for the treatment of PD [6] with detailed description of initial medication on early PD patients. In general, Chinese guideline is similar to the American guidelines except for a few minor aspects. The age divide for DA and LD as initial therapy in China is defined as 65 years while in America it is 70 years old.

\section{Statistical Analysis}

We compared the initial therapy and current drug utilization of the 136 PD patients. To estimate the effect of guideline on physicians' prescription, chi-square test was employed to compare the use of DA and LD as initial therapy. Given delayed effect of the guideline on physicians, January 2007 and January 2010 were defined as two-time dividing points. And patients' age divide was 65 years old. We compared the two age groups' use of DA and LD pre-2007, during 2007-2009 and after 2009 as initial treatment. In addition, we also compared the use of DA and LD between class 3-A hospitals and non-class 3-A hospitals by chi-square test. Furthermore, because non-ergot DA was much more expensive than LD, the impact of insurance policy on the use of DA and LD was studied. Non-ergot DA was covered by medical insurance since
July 2011. Then we compared the prescription of non-ergot DA before and after 2011 by chi-square test. If the requirements for chi square tests were not met, then Fisher's exact test was performed. All analyses were performed with SPSS (version 17.0).

\section{Results}

This study cohort consisted of 136 patients including 75 males and 61 females. The mean age was 69.68 years. Disease onset ranged from 32 to 84 years old with the mean age of 62.01 years old. Disease duration varied from 1-34 years with the mean length of 7.59 years. The mean value of Hoehn-Yahr stage at PD onset was 1.20. When the study was conducted, Hoehn-Yahr stage ranged from 1-5 with mean value of 2.09. Of all the patients, 22 (16.2\%) paid the medical expenses themselves, 84 (61.8\%) benefited from medical insurance reimbursement with average costs of RMB 318 per month, the remaining $30(22.0 \%)$ patients enjoyed public health service.

\section{Prevalence of Anti-Parkinsonism Drug (Apd) Use}

There was a relatively high proportion of APD users received LD and Benserazide. Among the 136 studied patients, 99 (72.8\%) patients used LD and Benserazide, 38 (27.9\%) received Carbidopa and LD, 44 (32.4\%) received pramipexole, 31 (22.8\%) received piribedil, 32 (23.5\%) took amantadine, 21 (15.4\%) took entacapone, 17 (12.5\%) took trihexyphenidyl, 29 (21.3\%) received selegiline, and 2 (1.5\%) received Rasagiline. A total of 116 (85.3\%) received at least one $\mathrm{LD}$ ( $\mathrm{LD}$ and Benserazide or Carbidopa and LD) while $55.9 \%$ of the studied patients received DA (pramipexole or piribedil). In Table 1, prescriptions for each medication are presented. Prevalence of initial prescription is as follows (Table 2): 58.1\% (79) received LD and Benserazide, ranking first among APD; 8.8\% (12/136) received Carbidopa and LD, 3.7\% (5/136) received pramipexole, 4.4\% (6/136) received piribedil, $17.6 \%$ (24/136) took amantadine, $18.4 \%(23 / 136)$ received trihexyphenidyl, and $10.3 \%$ $(14 / 136)$ received selegiline. LD was chosen as initial therapy by a total of $91(66.9 \%)$ patients, while only $11(8.1 \%)$ preferred DA as initial prescription.

Table 1: Percentage of pharmacotherapy in the 136 patients.

\begin{tabular}{|c|c|c|c|c|c|c|c|c|c|}
\hline & $\begin{array}{c}\text { LD and } \\
\text { Benserazide }\end{array}$ & $\begin{array}{c}\text { Carbidopa } \\
\text { and LD }\end{array}$ & Pramipexole & Piribedil & Amantadine & Entacapone & Trihexyphenidyl & Selegiline & Rasagiline \\
\hline Patients & 99 & 38 & 44 & 31 & 32 & 21 & 17 & 29 & 2 \\
\hline Percentage & $72.8 \%$ & $27.9 \%$ & $32.4 \%$ & $22.8 \%$ & $23.5 \%$ & $15.4 \%$ & $12.5 \%$ & $21.3 \%$ & $1.5 \%$ \\
\hline
\end{tabular}

Table 2: percentage of initial pharmacotherapy in the 136 patients.

\begin{tabular}{|c|c|c|c|c|c|c|}
\hline & $\begin{array}{c}\text { LD and } \\
\text { Benserazide }\end{array}$ & Carbidopa and LD & Pramipexole & Piribedil & Amantadine & Trihexyphenidyl \\
\hline Patients & 79 & 12 & 5 & 6 & 24 \\
\hline Percentage & $58.10 \%$ & $8.80 \%$ & $3.70 \%$ & $4.40 \%$ & $17.60 \%$ & 14 \\
\hline
\end{tabular}

\section{Effects of Publishing the Guideline on the Prescription} Choice

Table 3 summarizes the changes of LD and DA prescriptions before and after the guideline publication. No significant difference between LD and DA prescriptions was found among patients $>65$ years old before and after $2007(\mathrm{P}=0.290)$. In patients $<65$ years, initial therapy of LD and DA differed before 2007, during 20072009 and after $2009(\mathrm{P}=0.005) .4 .2 \%$ patients received DA as initial 
therapy before 2007, and the proportion significantly increased to

found between pre-2007 and 2007-2009. 42.9\% during 2010-2012 ( $\mathrm{P}=0.028$ ). However, no significance was

Table 3: DA and LD used as initial prescriptions before and after the publication of the guideline

\begin{tabular}{|c|c|c|c|c|c|c|}
\hline \multirow{2}{*}{$\begin{array}{c}\text { Age at First } \\
\text { Treatment }\end{array}$} & \multicolumn{6}{|c|}{ Proportions of DA and LD used as Initial Prescriptions } \\
\hline & Before 2007 & After 2007 & 2007-2009 & After2010 & Pearson $\mathrm{x} 2$ & $P$ valuea \\
\hline \multirow{2}{*}{$\geq 65$} & $1 / 19^{\mathrm{b}}$ & $6 / 35$ & & & 1.228 & 0.409 \\
\hline & $1 / 19$ & & $1 / 11$ & $5 / 24$ & 1.891 & 0.388 \\
\hline \multirow{5}{*}{$<65$} & $1 / 23\left(4.2^{\mathrm{c}}\right)$ & $3 / 14(17.6)$ & & & 2.054 & 0.290 \\
\hline & $1 / 23(4.2)$ & & $0 / 10$ & $3 / 4(42.9)$ & 10.644 & 0.005 \\
\hline & $1 / 23(4.2)$ & & $0 / 10$ & & 0.429 & 1.000 \\
\hline & $1 / 23(4.2)$ & & & $3 / 4(42.9)$ & 7.219 & 0.028 \\
\hline & & & $0 / 10$ & $3 / 4(42.9)$ & 5.204 & 0.051 \\
\hline
\end{tabular}

a) Comparison of the number of patients used DA and LD as initial prescriptions at different time-periods using chi-square test or the Fisher's exact test with one degree of freedom where applicable. Time periods including $<2007$ and $>2007$ or $<2007$, 2007-2009 and $>2009$.

b) Proportions of DA and LD utilization.

c) Percentage of DA utilization.

\section{The Influence of Hospital Class and Insurance Types on} Initial Prescriptions

Initial therapy of LD and DA were compared between Class3-A hospitals and non-Class3-A hospitals. A total of 104 patients were enrolled. 88 patients received initial prescription in Class-3A hospital among whom 76 received LD and 12 used DA. There were 16 patients receiving initial prescription in non-Class 3-A hospitals, among whom 15 received $L D$ and 1 received DA. Initial prescription of DA was not significantly different between Class 3-A hospitals (13.6\%) and non-Class 3-A hospitals (6.3\%) $(\mathrm{P}=0.686)$.

\section{Changes of Non-Ergot Da Prescription Before and After it was Covered by Medical Insurance}

It was not until 1/7/2011 that non-ergot DA (pramipexole and piribedil) were formally covered by medical insurance in Beijing. Among the 111 patients in our study before July 2011, 50 (45\%) patients received non-ergot DA (28 received pramipexole and 22 received piribedil). From the start of the survey to April 2012, the number of patients receiving non-ergot DA increased to 76 (44 received pramipexole and 32 received piribedil) with a proportion up to $55.9 \%$. However, no significant insurance-related differences were found.

\section{Discussion}

\section{Impact of the Publication of the Guideline on Initial Prescription}

In our study, the guideline did not significantly influence initial prescription in patients $\geq 65$ years old. The outcomes were under expectation since the chances of motor complication induced by LD remained relatively uncommon in old patients. The suggestion of LD prescription in patients $\geq 65$ years in the guideline was in line with medical practice before. The guideline dramatically promoted
DA utilization in young patients. In general, this statistic results were in agreement with the population-based study in France [7]. However, there existed delay in the implementation of the guideline because no significant differences were found between pre-2007 and 2007-2009. However, initial prescription differed significantly between pre-2007 and 2010-2012. The delay in the practice of guideline could be due to the lack of Chinese physicians' attention initially. Economy and medical insurance also may have influence on drug prescription. Increased influence of the guideline on Chinese physicians' practice since 2010 was mainly driven by Movement disorders and Parkinson's disease study group of Chinese Society of Neurology. Besides, the study group revised and republished the guideline in 2009 which may further enhance the influence of the guideline. It is likely that other factors such as insurance coverage, the publication of related clinical trials and the promotion of pharmaceutical manufactures also contribute to the phenomenon. The guideline improved the proportion of DA utilization in young patients. However, according to our study, a large number of patients $<65$ years received LD initially with a proportion of $63.9 \%$, while only $13.7 \%$ patients received DA as the initial therapy. This is consistent with surveys abroad [8-11]. It implied that only a minority of physicians prescribed APD based on the guideline and it took time to change clinical practice after the publication of the guideline.

\section{The Influence of Hospital Level and Medical Insurance on Initial Prescription}

No significant influence of hospital level and the types of medical insurance on initial prescription were found. Possible reasons were as follows: first of all, daily clinical practice is compliant with the principle of PD medication, both physicians and patients aimed at controlling symptom. Secondly, most patients from class 2-A hospitals we surveyed were from Pinggu traditional 
Chinese medical hospital where regulatory outpatient service was offered by the physicians from Beijing Hospital of the Ministry of Health. The offered outpatient service can largely improve diagnostic and treatment level. However, some other class 2-A hospitals may offer poor service and patients from those hospitals may not be included in our study. Among the 20 patients who were free of medical insurance, 10 patients were from other provinces. However, these 10 patients had the ability to afford medical costs and had high drug compliance. Finally, another limitation of the presented study should be taken into account since our findings were based on a relatively small number of subjects. Especially, the proportions of initial prescriptions from class 2-A hospitals and patients free of medical insurance were quite small. Two DA (pramipexole and piribedil) started to be covered by medical insurance since 1/7/2011 in Beijing which drove DA utilization modestly $(\mathrm{P}=0.098)$. We then performed Fisher exact test with a $\mathrm{p}$ value of 0.058 . Significant difference may be found when a large number of patients are enrolled. To some extend our study shows that drug compliance and economic burden are needed to be taken into consideration when physicians prescribe medications in China. Moreover, the guideline promotion needs to be supported by medical insurance to some extent.

\section{References}

1. J Satheesh Kumar, P Bhuvaneswari (2012) Analysis of Electroencephalography (EEG) and its categorization A Study. Procedia Engineering 38: 2525-2536.

2. Tanja Schultz (2017) Bio signal Based Spoken Communication: A Survey. ACM Transactions on audio, speech, and language processing IEEE 25(12).

3. Sandhya Joshi, Deepa Shenoy (2010) Classification of Alzheimer's disease and Parkinson's Disease by Using Machine Learning and Neural Network Methods. Second International Conference on Machine Learning and Computing

4. A M Ardi Handojoseno, James M Shine (2012) The detection of Freezing of Gait in Parkinson's disease patients using EEG signals based on Wavelet Decomposition. $34^{\text {th }}$ Annual International Conference.

5. Jonathan Weyhenmeyer (2014) Muscle artifacts in single trail EEG data distinguish patients with Parkinson's disease from healthy individuals. $36^{\text {th }}$ Annual International Conference of the IEEE Engineering in Medicine and Biology Society.

6. Quynh Tran Lya (2016) Detection of gait initiation failure in Parkinson's disease patients using EEG signals. $38^{\text {th }}$ Annual International Conference of the IEEE Engineering in Medicine and Biology Society.

7. Quynh Tran Ly (2016) Identifying montages that best detect the electroencephalogram power spectrum alteration during freezing of gait in Parkinson's disease patients. $38^{\text {th }}$ Annual International Conference of the IEEE Engineering in Medicine and Biology Society (EMBC) pp 60946097.

8. A Maurer, S Hanrahan, J Nedrud (2016) Suppression of Neurostimulation Artifacts and Adaptive Clustering of Parkinson's Patients Behavioral Tasks using EEG. 50 ${ }^{\text {th }}$ Asilomar Conference on Signals, Systems and Computers.

9. Quynh Tran Lya, AM Ardi Handojoseno (2017) Detection of Turning Freeze in Parkinson's Disease based on S-transform Decomposition of EEG signals. Annual International Conference of the IEEE Engineering in Medicine and Biology Society pp3044-3047.
10.AM Ardi Handojoseno, James M Shine (2013) Using EEG Spatial Correlation, Cross Frequency Energy and Wavelet Coefficients for the prediction of Freezing of Gait in Parkinson's disease patients. $35^{\text {th }}$ Annual International Conference EMBS IEEE pp 4263-4266.

11. AM Ardi Handojoseno (2015) An EEG Study of Turning Freeze in Parkinson's disease Patients: The Alteration of Brain Dynamic on the Motor and Visual Cortex. Annual International Conference of the IEEE Engineering in Medicine and Biology Society, pp. 6618-6621.

12. Max A Little (2009) Suitability of Dysphonia Measurements for Telemonitoring of Parkinson's disease. Transactions on Biomedical Engineering.

13. Resul Das (2009) A comparison of multiple classification methods for Diagnosis of Parkinson disease. Expert Systems with Applications 37(2): 1568-1572.

14. Hui Ling Chen (2012) An efficient diagnosis system for Detection of Parkinson's disease using fuzzy k nearest neighbor approach. Expert Systems with Applications 40(10): 263-271.

15. Michal Vadovský, JánParali (2017) Parkinson's Disease patients Classification based on the speech signals 15th International Symposium on Applied Machine Intelligence and Informatics (SAMI).

16. Betul Erdogdu Sakar, M Erdem Isenkul (2013 ) Collection and Analysis of a Parkinson Speech Dataset with Multiple Types of Sound Recordings IEEE- Hananel Hazan et al. Early diagnosis of Parkinson's disease via machine learning on speech data. $27^{\text {th }}$ Convention of Electrical and Electronics Engineers-IEEE 17(4): 828-834.

17. Hananel Hazan (2012) Early diagnosis of Parkinson's disease via machine learning on speech data- $27^{\text {th }}$ Convention of Electrical and Electronics Engineers- IEEE.

18. Spriha Chandrayan (2017) Selection of Dominant Voice Features for Accurate Detection of Parkinson's disease. $3^{\text {rd }}$ International Conference on Bio signals, images and instrumentation-IEEE.

19. Laetitia Jeancolas (2017) Automatic Detection of Early Stages of Parkinson's Disease through Acoustic Voice Analysis with Mel-Frequency Cepstral Coefficients. International Conference on Advanced Technologies for Signal and Image Processing (ATSIP).

20. M Hariharan (2014) A new hybrid intelligent system for accurate detection of Parkinson's disease. Comput Methods Programs Biomed 113(3): 904-913.

21. ZhennaoCai (2017) A New Hybrid Intelligent Framework for Predicting Parkinson's Disease. IEEE.

22. K Lopez de Ipna (2012) New approaches for Alzheimer Disease Diagnosis based on Automatic Spontaneous Speech Analysis and Emotional Temperature. $4^{\text {th }}$ International work shop pp 407-414.

23. Vassilis Baldas (2011) Early Diagnosis of Alzheimer's Type Dementia Using Continuous Speech Recognition.

24. Ali Khodabakhsh (2014) Detection of Alzheimer's disease using prosodic cues in conversational speech. $22^{\text {nd }}$ Signal Processing and Communications Applications Conference (SIU).

25. Vassili killiadou (2004) Clinical psychoacoustics in Alzheimer's disease central auditory processing disorders and speech deterioration. A Review. Annals of General Hospital Psychiatry 2(1): 12.

26. Alxendra Koing (2015) Automatic speech analysis for the assessment of patients with pre-dementia and Alzheimer's disease. Alzheimer's \& Dementia: Diagnosis, Assessment \& Disease Monitoring 1(1): 112-124.

27. Tyler Staudinger (2011) Analysis of Complexity Based EEG Features for the Diagnosis of Alzheimer's Disease. Conference of the IEEE Engineering in Medicine and Biology Society pp 2033-2036.

28. Lineu Corrêa Fonseca (2011) Quantitative electroencephalography power and coherence measurements in the diagnosis of mild and moderate Alzheimer's disease. Neuro Psiquiatr. 69: 297-303. 
29. Mohamed Elgendi (2011) Optimization of EEG frequency bands for improved diagnosis of Alzheimer disease. Conference of the IEEE Engineering in Medicine and Biology Society. Pp. 6087-6091.

30. Davide Vito Moretti (2014) The Contribution of EEG to the Diagnosis of Dementia. J. Biomedical Science and Engineering, pp. 546-562.

31. Noor Kamal Al Qazzaz (2014) Role of EEG as Biomarker in the Early Detection and Classification of Dementia. The Scientific World Journal, pp. 16.
32. Nilesh N Kulkarni (2017) Use of Non-linear Complexity features for EEG based dementia and Alzheimer's disease Diagnosis

33. Carlos Gómez (2016) Analysis of spontaneous EEG activity in Alzheimer's disease using cross-sample entropy and graph theory. Conference of the IEEE Engineering in Medicine and Biology Society, pp. 2830-2833.

\section{(c) (1) \\ This work is licensed under Creative Commons Attribution 4.0 License}

To Submit Your Article Click Here:

Submit Article

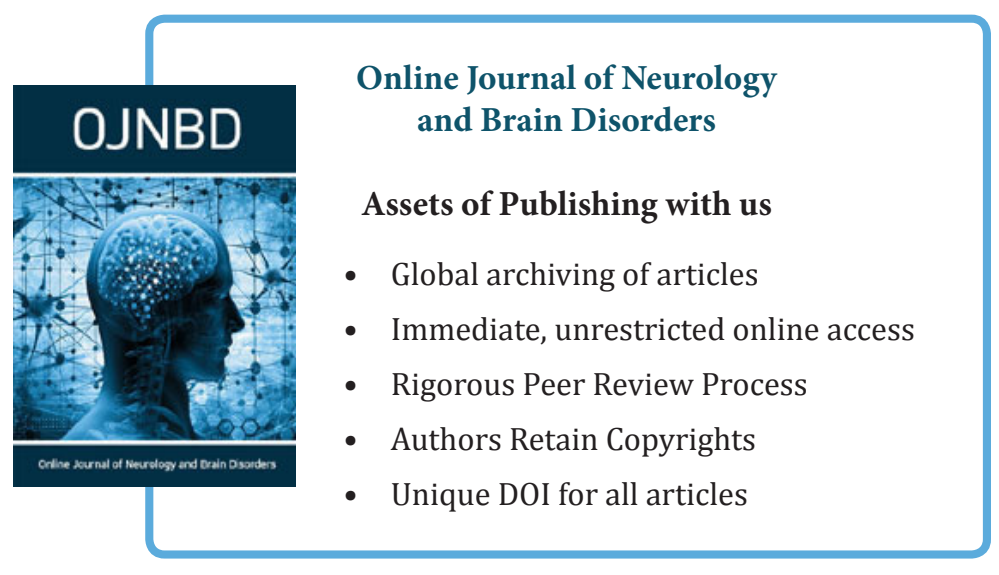

